Collection SFN 7 (2007) 241-260

(c) EDP Sciences, Les Ulis

DOI: 10.1051/sfn:2007025

\title{
Nuclear spin contrast in structural biology
}

\author{
H. Stuhrmann ${ }^{1}$
}

${ }^{1}$ GKSS Forschungszentrum, Geesthacht, Germany and Institut de Biologie Structurale Jean-Pierre Ebel, CEA/CNRS/UJF Grenoble, France

\begin{abstract}
Nuclear spin contrast is observed at its best with polarized neutrons. In the presence of paramagnetic centers nuclear spins are polarized by the method of dynamic nuclear spin polarization (DNP). Nuclear spin contrast is strongest with polarized protons. Proton spin contrast in some sense extends the range of magnetic neutron scattering to very dilute paramagnets, hence its interest in structural biology
\end{abstract}

\section{INTRODUCTION}

Nearly all the work done with neutrons in biology is based on the extraordinary properties of the naturally abundant hydrogen and its heavier isotope deuterium. In terms of its neutron scattering amplitude, deuterium behaves very much like the other biologically most frequent nuclei, carbon, oxygen, whereas normal hydrogen is 'exotic', with an amplitude about half the size of deuterium but negative. There is more to hydrogen as has been noticed by Hayter et al. [1]. The neutron scattering amplitude of hydrogen varies strongly with the relative orientation of the neutron spin interacting with the proton spin. Thus the best conditions for a neutron scattering experiment are realized if both the neutron beam and the nuclear spins of the sample are polarized [2]. 'Ideal' neutron scattering experiments using polarized neutrons and polarized nuclei were very rare for a long time [3,4]. In fact, their renaissance started only in the mid 1980s thanks to an extremely fruitful co-operation with particle physicists [5-8]. First results obtained at the ILL have been reported by van den Brandt et al. [9] This paper will focus on those experiments of polarized neutron scattering which paved the way to nuclear spin contrast variation in biological structure research.

\section{NEUTRON SCATTERING THEORY}

Neutrons interact with matter either through their nuclear interactions with the atomic nucleus or magnetically of the atoms carry an electric moment. Detailed treatments can be found in textbooks on neutron scattering. Rigorous treatments of neutron scattering from polarized nuclei are given by Abragam and Goldman [10], des Cloizeaux and Jannink [2], Glättli and Goldman [11] and Leymarie [12].

\subsection{Spin dependance of nuclear interaction}

Let an isotope with spin $I(I \neq 0)$ associated with its operator, $\mathbf{I}$, and a neutron spin $;$ be associated with its operator, $\mathbf{s}$. The interaction, and hence the resulting scattering length will depend on the total angular momentum $\mathbf{J}=\mathbf{I}+\mathbf{s}$ of the neutron-isotope system. Taking into account the rules for addition of angular moments, there are two different values of $\mathbf{J}$ that go together with two different scattering lengths

$$
b=b_{ \pm} \longleftrightarrow \mathbf{J}_{ \pm}=\mathbf{I} \pm 1 / 2
$$

Each of these states has $2 \mathrm{~J}+1$ substates. It is a convenient practice to express this dependence of the scattering length as a function of the operators $\mathbf{I}$ and $\mathbf{s}$ :

$$
b=b_{0}+b_{n} \mathbf{I} . \mathbf{s}
$$


Using

$$
2 \mathbf{I} . \mathbf{s}=\mathbf{J}^{2}-\mathbf{I}^{2}-\mathbf{s}^{2}=J(J+1)-I(I+1)-3 / 4
$$

the comparison of equations 2.2 and 2.3 yields

$$
\begin{aligned}
& J_{+}=I+1 / 2 \longrightarrow 2 \mathbf{I} . \mathbf{s}=I \longrightarrow b_{0}+I b_{n} / 2=b_{+} \\
& J_{-}=I-1 / 2 \longrightarrow 2 \mathbf{I} . \mathbf{s}=-(I+1) \longrightarrow b_{0}-(I+1) b_{n} / 2=b_{-}
\end{aligned}
$$

$b_{0}$ and $b_{n}$ are obtained as

$$
\begin{aligned}
& b_{0}=\left[(I+1) b_{+}+I b_{-}\right] /(2 I+1) \\
& b_{n}=2\left(b_{+}-b_{-}\right) /(2 I+1)
\end{aligned}
$$

The following table contains the scattering length of some isotopes most frequently occurring in soft condensed matter.

Table 1. Neutron scattering lengths $b_{0}$ and $b_{n}$ of selected isotopes in units of $10^{-12} \mathrm{~cm}$ in comparison with the X-ray scattering lengths $b_{x}$.

\begin{tabular}{c|c|c|c|c}
\hline Isotope & Spin & $b_{0}$ & $b_{n}$ & $b_{x}$ \\
\hline${ }^{1} H$ & & -0.374 & 5.824 & 0.28 \\
$\mathrm{H}-2$ & 1 & 0.667 & 0.570 & 0.28 \\
$\mathrm{C}-12$ & 0 & 0.665 & 0 & 1.7 \\
$\mathrm{C}-13$ & $1 / 2$ & 0.62 & -0.12 & 1.7 \\
$\mathrm{~N}-14$ & 1 & 0.94 & 0.28 & 2.0 \\
$\mathrm{O}-16$ & 0 & 0.580 & 0 & 2.2 \\
\hline
\end{tabular}

Several remarks on the spin dependence may be useful. First, it is not due to the magnetic moments of two interacting spins. There is a difference of several orders of magnitude between these two phenomena [11]. Moreover, it is easily recognized that nuclei with large $b_{n}$ like ${ }^{1} H$ are ideally suited for experiments of nuclear spin dependent polarized neutron scattering. The absorption of neutron by soft condensed matter is small.

\subsection{Coherent and incoherent scattering}

The intensity scattered by a sample is made up of one part that is called coherent and another part that is called incoherent. Coherent scattering arises from scattering centers with definite spatial correlation or order. This is the useful signal as it gives access to the spatial correlation between scattering centers and hence to the microscopic structure of the sample. As for the incoherent scattering, two sources are relevant: structural disorder and quantum processes that destroy the coherence of scattering. In both cases the incoherent scattering is characterized by its independence from the momentum transfer $\mathbf{Q}$.

For a collection of $\mathrm{n}$ atoms at $\mathbf{r}_{n}$, without spin, and in the absence of spatial correlations of the fluctuations of $b$ the differential cross section of neutron scattering is

with

$$
\begin{aligned}
d \sigma / d \Omega & =n b_{i n c o h .}^{2}+b_{c o h .}^{2} \Sigma_{k, l} \exp \left(i \mathbf{Q}\left(\mathbf{r}_{l}-\mathbf{r}_{k}\right)\right) \\
b_{\text {incoh }}^{2} & \left.\left.=\left\langle b_{k}^{2}\right\rangle-\left\langle b_{k}\right\rangle\right\rangle^{2}, b_{c o h}^{2}=\left\langle b_{k}\right\rangle\right\rangle^{2}
\end{aligned}
$$

Now let us consider a collection of atoms of the same isotope with spin I. According to equ. 2.3 and knowing that s.I $=s_{z} I_{z}+\left(s_{+} I_{-}+s_{-} I_{+}\right)$, the scattering length can be written as follows:

$$
b=b_{0}+b_{n} s_{z} I_{z}+b_{n}\left(s_{+} I_{-}+s_{-} I_{+}\right)
$$


This expression of $\mathrm{b}$ allows to rearrange the scattering process into two groups:

(a) a process without spin flip with the scattering length $b_{0}+b_{n} s_{z} I_{z}$, which includes coherent scattering and a part of incoherent scattering due to the disorder of spin in a not completely polarized sample.

(b) a process with spin flip with the scattering length $b_{n}\left(s_{+} I_{-}+s_{-} I_{+}\right)$, which is entirely incoherent. There is no interference between different nuclear spins because their final states are not the same.

Taking into account the rules of commutation between spin operators, it can be shown that for two atoms $\mathrm{k}$ and 1 the following relation holds

$$
\begin{aligned}
& b_{k}^{2}=b_{0}^{2}+2 b_{0} b_{n} \mathbf{s . \mathbf { I } _ { k }}+b_{n}^{2}\left(I(I+1) / 4-\mathbf{s . \mathbf { I } _ { k }} / 2\right)
\end{aligned}
$$

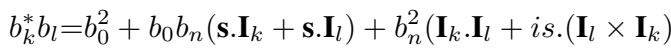

Assuming that there is no correlation between $\mathbf{I}_{k} . \mathbf{I}_{l}$ and $\mathbf{r}_{i}-\mathbf{r}_{j}$ one obtains an expression of the cross section that is analogous to equ. 2.7

$$
\frac{d \sigma}{d \Omega}(\mathbf{Q})=\sum_{k}\left\langle b_{k}^{2}+\sum_{k \neq l}\left\langle b_{k}^{*} b_{l}\right\rangle\left\langle\exp \left(i \mathbf{Q} \cdot\left(\mathbf{r}_{l}-\mathbf{r}_{k}\right)\right)\right\rangle\right.
$$

Finally, it is assumed that the nuclei and the neutrons are polarized with respect to the same axis, $\mathbf{e}_{z}$. With the polarization $\mathrm{P}$ and $\mathrm{p}$ of the nuclei and neutrons, respectively, defined as

$$
\begin{aligned}
\left\langle\mathbf{I}_{k}\right\rangle \mathbf{e}_{z} & =P I \\
\langle\mathbf{s}\rangle e_{z} & =p / 2
\end{aligned}
$$

one obtains

$$
\left\langle b_{k}^{2}\right\rangle=b_{0}^{2}+b_{0} b_{n} I p P+b_{n}^{2}[I(I+1)-p P I],\left\langle b_{k}^{*} b_{l}\right\rangle_{k \neq l}=b_{0}^{2}+b_{0} b_{n} I p P+b_{n}^{2} I^{2} P^{2}
$$

In the same way as it is practice for isotopic incoherence, 2.16 can be rewritten as follows,

$$
\frac{d \sigma}{d \Omega}(\mathbf{Q})=\sum_{k=l}\left(\left\langle b_{k}^{2}\right\rangle-\left\langle b_{k}^{*} b_{l}\right\rangle_{k \neq l]}+\left\langle b_{k}^{*} b_{l}\right\rangle_{k \neq l)} \sum_{k, l} \exp \left(i \mathbf{Q} \cdot\left(\mathbf{r}_{l}-\mathbf{r}_{k}\right)\right)\right.
$$

and one obtains finally

with

$$
\frac{d \sigma}{d \Omega}=\frac{d \sigma}{d \Omega}_{\text {incoh. }}+b_{\text {coh. }}^{2} \sum_{k, l} \exp \left(i Q\left(r_{l}-r_{k}\right)\right)
$$

and

$$
\begin{aligned}
\frac{d \sigma}{d \Omega}_{\text {incoh. }} & =\frac{n b_{n}^{2}}{4}\left[I(I+1)-p P I-P^{2} I^{2}\right] \\
b_{c o h}^{2} & =b_{0}^{2}+b_{0} b_{n} I p P+\frac{b_{n}^{2} I^{2} P^{2}}{4}
\end{aligned}
$$

The largest incoherent scattering is encountered with $\mathrm{pP}=-1$ (spins antiparallel). There is no spin incoherent scattering for $\mathrm{pP}=1$ (spins parallel). In fact, at $\mathrm{pP}=1$, scattering with spin flip is impossible because there is no spin disorder. On he other hand, for $\mathrm{pP}=-1$ there is still no spin disorder, but the probability of scattering with spin flip is largest (Figure 1). 


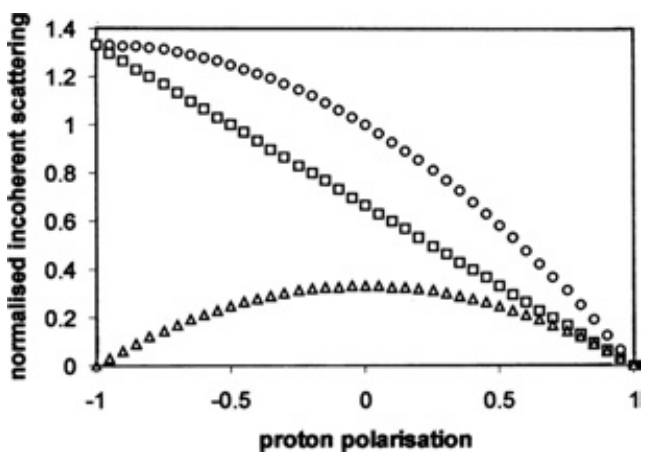

Figure 1. Incoherent scattering $(\bigcirc)$ with $(\square)$ and without $(\triangle)$ change of the spin state of neutron and proton, normalized to incoherent scattering at $\mathrm{P}=0$.

Most biological applications of neutron scattering rely on isotopic substitution of hydrogen, ${ }^{1} \mathrm{H}$, by deuterium. The change of the scattering length is $1.04 \times 10^{-12} \mathrm{~cm}$. A much more important change of the scattering length by $2.9 \times 10^{-12} \mathrm{~cm}$ can be achieved by polarized neutron scattering from polarized protons. When the proton spin is opposite to the spin of the incident neutron ( $\mathrm{pP}=-1)$, the scattering length of the proton becomes strongly negative and thus even more different from the scattering lengths known from other nuclei. For structural studies aiming at the location of hydrogen, this situation is very attractive. Inspection of Figure 1 tells us that at the same time incoherent scattering becomes largest. Structural studies will have to rely on a partial deuteration of the sample, the degree of deuteration depending on what is desirable or possible.

\subsection{Nuclear spin contrast}

In contrast to the method of isotopic substitution, which requires preparation of several samples, a single sample will be sufficient for contrast variation through nuclear spin polarization. Using a completely polarized neutron beam, the variation of the scattering length with nuclear polarization, $\mathrm{P}$, is described in a similar way as is done for isotopic substitution.

$$
\left\langle b_{k}\right\rangle=b_{0}+b_{n} I p P
$$

Hence, the coherent scattering length of the hydrogen isotopes is

$$
\begin{aligned}
& b_{H}=(-0.374 \pm 1.456 P(H)) 10^{-12} \mathrm{~cm} \\
& b_{D}=(+0.667 \pm 0.27 P(D)) 10^{-12} \mathrm{~cm}
\end{aligned}
$$

where $\mathrm{P}(\mathrm{H})$ and $\mathrm{P}(\mathrm{D})$ are the polarization of protons and deuterons, respectively. The sign \pm refers to the polarization of the incident neutron beam, which is assumed to be $p= \pm 1$. While almost completely polarized neutron beams of high intensity are obtained routinely, a high nuclear polarization is achieved less readily.

\subsection{Nuclear spin polarization}

Magnetic moments can be oriented in space by putting them in a magnetic field. This also applies to nuclei with a magnetic moment, i.e. nuclei with spin $I \neq 0$. Nuclei are said to be oriented when the populations $\mathrm{w}(\mathrm{m})$ of the magnetic substates with quantum numbers $\mathrm{m}$ are not all equal. The degree of orientation can be described by orientation parameters of increasing order, $f_{1}, f_{2}, \ldots$ [13]. The first parameter is called polarization and is expressed in the population numbers, $\mathrm{w}(\mathrm{m})$, as $f_{1}=\sum m w(m) / I$. 
As the magnetic moment of nuclear spins is very small, the natural polarization even under conditions of a strong magnetic field and low temperatures remains nearly negligible. In order to obtain a higher nuclear polarization, as it is needed for polarized targets of particle physics and for experiments of nuclear spin contrast variation, the method of dynamic nuclear polarization (DNP) is used. In this case, the temperature of the nuclear spin system is different from that of the lattice. Such methods have been shown to exist by Overhauser [14] in conducting materials and be Abragam [15] in insulators.

As the constituents of living cells, proteins, nucleic acids, and lipids are non-conducting solids, we are interested in the method of Abragam. In this case, the nuclear spins of these materials are polarized in the presence of paramagnetic centers that have been added in a small amount.The temperature is kept between $0.1 \mathrm{~K}$ and $1 \mathrm{~K}$, and a high magnetic field is used. Various methods of DNP exist that differ in the way how the polarization of the electronic spin system is transferred to the nuclear spin system [16].

The macroscopic aspects of DNP are well understood in the frame of the spin temperature theory [16]. The thermodynamic model assigns heat reservoir models to various degrees of freedom of the electronic and nuclear spin systems that are coupled via mutual and external interactions. The mechanism of DNP then is described as a two step process: the cooling of the non-Zeeman reservoir by a nonsaturating microwave field and the subsequent transfer of entropy from the nuclear Zeeman system via thermal mixing. It is assumed that this transfer is efficient, so that the spin temperatures of both systems will become equal. An upper limit of the achievable nuclear polarization can be given [17]. However even a refined model that takes into account a non-ideal cooling process considering an electron spin system with hyperfine interactions and g-factor anisotropy [18] yields too optimistic values. In practice, very different maximum bulk nuclear polarizations are observed for closely related materials, indicating the importance the second step of DNP, the thermal mixing. The magnitude of the mixing is strongly influenced by the microscopic structure of the material and in particular the nuclear spins close to the unpaired electrons [19-21]

\subsection{Nuclear magnetic resonance}

Methods of NMR spectroscopy are important tools for the characterization and subsequent modification of dynamically polarized targets:

(a) The polarization of each non-spinless isotope is determined using NMR

(b) The polarization of each isotopic spin can be reversed or destroyed selectively

(c) For each isotope, the just mentioned selectivity also applies to nuclear spins at different distances from a paramagnetic center. Proton NMR from a dynamically polarized target containing a chromium-(V) complex, EHBA-Cr(V), in perdeuterated butanol may serve as an example (Figure 2).

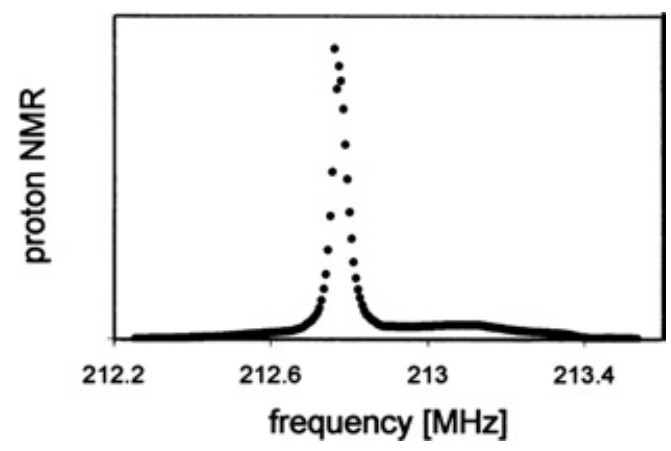

Figure 2. NMR spectrum of EHBA-Cr(V) in perdeuterated butanol (0.98). B = 5 T. From Niinikoski [22]. 
A narrow peak is observed on the top of a much broader asymmetric profile. The narrow peak is interpreted as arising from the residual unsubstituted protons of the solvent. The broad line most likely comes from the protons close to the paramagnetic center, i.e. the 20 protons of the EHBA-Cr(V) molecule. The origin of the line shape of the broad line is a combination of dipolar and hyperfine interactions with the electron spin of the molecule. Clearly, the central peak of proton NMR is most easily determined. The integrated intensity of the central peak is a measure of the bulk proton polarization. The signal is calibrated at some standard conditions, e.g. at $\mathrm{B}=2.5 \mathrm{~T}$ and $\mathrm{T}=1 \mathrm{~K}$, where the proton polarization is known to be 0.0025 .

The method of adiabatic fast passage (AFP) is used for reversal of the nuclear spin polarization [23]. A radio frequency sweep will affect only those spins the resonance of which falls into the selected frequency interval, without changing the state of those protons that have a different resonance frequency. Hence an rf sweep over the central proton NMR peak will reverse the polarization of the bulk protons, whereas the 'close' protons are much less affected.

\section{EXPERIMENTAL TECHNIQUES}

Facilities for DNP meeting the requirements of neutron diffraction are run at various neutron scattering laboratories, e.g. LLB Saclay, GKSS Geesthacht, PSI Villigen.

The common features are the following:

1. The sample is cooled by liquid helium

2. For temperatures below $1 \mathrm{~K}$, the ${ }^{4} \mathrm{He}$ bath of the sample cell id coupled to the mixing chamber of a dilution refrigerator, the ${ }^{3} \mathrm{He} /{ }^{4} \mathrm{He}$ mixture of which reaches temperatures slightly below $0.1 \mathrm{~K}$.

3. The liquid helium consumption is moderate : between 1 and $2 \mathrm{dm}^{3} / \mathrm{h}$

4. The magnet is designed so as to allow for a large solid angle for the scattered neutrons

5. The sample exchange time is short : less than half an hour for $1 \mathrm{~K}$ cells, less than half a day for $0.1 \mathrm{~K}$ cells

6. The sample volume is slightly less than $1 \mathrm{ml}$. The size of the platelets is $15 \times 15 \times 3 \mathrm{~mm}^{3}$.

\subsection{Cryogenics}

Temperatures well below $1 \mathrm{~K}$ are obtained by a dilution refrigerator. A relatively powerful refrigerator, which can cope with a heat load of $0.3 \mathrm{~mW}$ at $0.1 \mathrm{~K}$, has been adapted by CERN, Geneva, to the requirements of neutron scattering. This set up is installed at the neutron reactor of GKSS, Geesthacht [24].

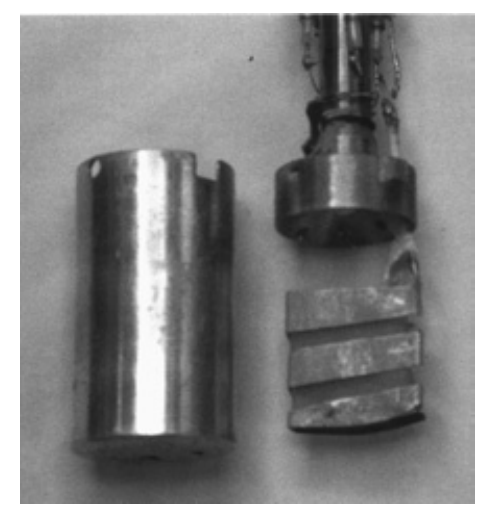

Figure 3. NMR coil, guide, and high frequency cavity of PSI. 
Temperatures of $1 \mathrm{~K}$ are readily obtained by rapid evaporation of liquid helium. A polarized target system of the Paul-Scherrer-Institut (PSI) [25] based on that cryogenic technique has been modified for neutron scattering experiments $[25,26]$. A $3.5 \mathrm{~T}$ split coil, wound on an aluminium former, is attached to the bottom of a liquid helium vessel. A stainless steel tube of $49 \mathrm{~mm}$ diameter with an aluminium end cap runs axially through the helium bath an then in vacuum down to the center of the magnet. It accommodates a continuous flow helium refrigerator insert with a top loading sample holder device. The top loading sample holder is approximately $1 \mathrm{~m}$ long and built around a cylindrical waveguide ending in an aluminium cavity for samples of up to $17 \mathrm{~mm}$ diameter (Figure 3 ). The system has a cooling power of $10 \mathrm{~mW}$ at $1 \mathrm{~K}$.

The direction of the neutron beam coincides with that of the magnetic field inside the coil. The large opening angle of the magnet allows the measurement of neutron scattering intensity over a large solid angle defined by $\pm 45^{\circ}$ for both horizontal and vertical direction. Using wavelengths of $4.5 \AA$ the structural resolution is limited to $6 \AA$. Tilting the axis of the magnet with respect to the neutron beam would increase the structural resolution, e.g. for single crystal diffraction, or allow special studies, e.g. on the supposed asymmetry of selectively polarized polarization domains. The set up is easily moved to other places. It has been operated successfully at the instruments SANS-1 at PSI and D22 at the ILL.

\subsection{The microwave system}

The high frequency system consists of a source, a waveguide and a multimode cavity. The high frequency generator of $70 \mathrm{GHz}$ used at LLB and at GKSS is a carcinotron. The power of the carcinotron is several watts. The frequency range controlled by the power supply as large enough to allow a frequency jump of $300 \mathrm{MHz}$ in a few microseconds which is needed to change the direction nuclear polarization by DNP [12]

The PSI microwave system differs both in the number of sources and in the frequencies used. Two IMPATT diodes of $100 \mathrm{~mW}$ output power, tuned to frequencies corresponding to positive and negative polarization by DNP, respectively ( 97.0 and $97.5 \mathrm{GHz}$ ), can be connected alternately to the sample cavity by an electro-mechanical wave guide switch. It takes $165 \mathrm{~ms}$ to swap the frequency, most of the time $(150 \mathrm{~ms})$ being used for the actual rotation with neither source connected. A status signal is set after each correct execution.

\subsection{The NMR and rf irradiation system}

The NMR signal is detected by a continuous wave (cw) spectrometer. It consists of a rf source, a NMR coil (Figure 3), a detection system called Q-meter, and a digitization unit. The system is sensitive to a change of the impedance of the sample due to the polarization of its nuclear spins. More precisely, the polarization is obtained as the integral over the imaginary part of the susceptibility [27] For proton NMR with magnetic fields of $2.5 \mathrm{~T}$ (GKSS and LLB) and $3.5 \mathrm{~T}$ (PSI) the system is operated at $106 \mathrm{MHz}$ and $150 \mathrm{MHz}$, respectively. Through a coaxial switch inserted in the (3/2) $\lambda$ cable connecting the NMR coil with the Q-meter, a separate dedicated rf system can be connected to the coil. It is used to manipulate separately the nuclear spin systems of the sample, e.g. to perform AFP polarization reversals [28] or to destroy the nuclear polarization.

\subsection{Samples and sample preparation}

In most cases DNP has been performed in the presence of a chromium-(V) complex, $\mathrm{C}_{12} \mathrm{H}_{20}-$ $\mathrm{CrO}_{7} \mathrm{Na} \cdot \mathrm{H}_{2} \mathrm{O}$ [29], dissolved in a mixture of glycerol and water (11/9). EHBA-Cr(V) is very soluble in polar solvents. It is stable at acid $\mathrm{pH}$, and decomposes fairly rapidly at $\mathrm{pH} 7$ with a half time of a few minutes and instantaneously at still higher $\mathrm{pH}$. Some biological macromolecules, like ribosome solutions, 
require $\mathrm{pH}$ values close to $\mathrm{pH} 7$. This imposes some rules on the preparation of the sample. Glassy slabs of $3 \mathrm{~mm}$ thickness were obtained by injecting the solution into a copper mould cooled to $77 \mathrm{~K}$. This is done in a dry nitrogen atmosphere.

The biradical discussed below, is easily dissolved in organic solvents, like toluene. No well defined platelets with a regular shape can be obtained by the procedure described above. In this case polystyrene is added and the solvent is evaporated.The biradical molecules then are embedded in a polystyrene matrix.

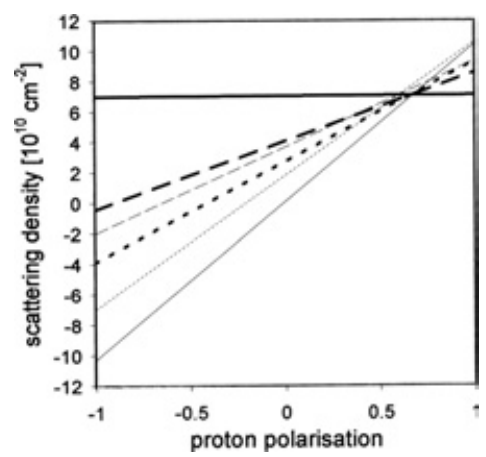

Figure 4. Variation of the scattering density of RNA and protein and of their protiated and deuterated solvent (glycerol/water, 1/1). Solid line : solvent, dashed line : RNA, dotted line : protein. RNA and protein in a deuterated solvent and the deuterated solvent itself are boldfaced.

\section{STATIC NUCLEAR SPIN CONTRAST}

By static nuclear spin contrast we mean stable (or stabilized) nuclear contrast over a longer period of time [12]. This approach is suitable for structural studies. Let $U(\mathbf{Q})$ be the structure amplitude of the unpolarized sample and $V(\mathbf{Q})$ the structure amplitude due to nuclear polarization then the intensity of coherent scattering from randomly oriented particles is [10]

$$
I(Q)=\left\langle|U(\mathbf{Q})|^{2}+2 p \operatorname{Re}\left[U(\mathbf{Q}) V^{*}(\mathbf{Q})\right]+|V(\mathbf{Q})|^{2}\right\rangle
$$

Experiments of polarized neutron scattering from proton spin-polarized macromolecules in solution were started in the mid-1980s at three different places, at the National Laboratory for High Energy Physics(KEK), Japan, at the Laboratoire Léon Brillouin, Saclay, France, and at the GKSS Research Center, Geesthacht, Germany. Knop et al. [5] published the first results of polarized neutron scattering from polarized targets of biological origin. Similar results were reported by Koghi et al [7] on crown ethers. In 1989, Glättli et al. [8] published the first results of neutron scattering from polarized protons in polymers.

The variation of the scattering density of RNA and proteins with proton polarization in a deuterated and in a non-deuterated solvent is shown in Figure 4. The variation of contrast is stronger in a deuterated solvent. The real argument in favor of a deuterated solvent is the low incoherent scattering intensity and hence the low attenuation of the neutron beam. Corrections for incoherent background and absorption then are small.

Most of the macromolecules studied so far have been specifically deuterated, i.e. some small region of interest inside the macromolecule has its protons replaced by deuterons [30]. In this case, the fraction of protiated material remains important. For a more drastic reduction of incoherent scattering the inverse isotopic substitution is preferred. The isotope ${ }^{1} H$ is substituted by ${ }^{2} H(=D)$ in the whole macromolecule except in those regions that for some reasons are of interest. Note that the native contrast of RNA and proteins is matched by nearly the same spin contrast around $\mathrm{P}=0.65$. Hence, proton 
spin polarization hardly distinguishes between RNA and proteins (Figure 4), whereas solvent contrast variation in $\mathrm{H}_{2} \mathrm{O} / \mathrm{D}_{2} \mathrm{O}$ mixtures does. The gain factor with respect to native contrast is between 2 and 3 . As all measurements of spin contrast variation are performed with one and the same sample, systematic errors are minimized.

\subsection{The ribosome}

The ribosome is a multi-component ribonucleo-protein complex that translates the genetic information provided by the messenger RNA into functional polypeptide chains, namely proteins. During protein synthesis several RNA ligands join the ribosome to form the functional complex, the mRNA, and the transfer RNA. The mRNA brings the genetic information to the ribosome, where tRNAs are present to interpret the codon sequence of the mRNA in terms of an amino acid sequence in the growing peptide. Data derived from cross linking studies at that time have led to the proposal of conflicting models for tRNA positions on the ribosome because of uncertainties in the spatial assignment of ribosomal components, hence the interest in neutron scattering techniques.

The functional complex of the ribosome $(\mathrm{M}=2300 \mathrm{kD})$ including both the small and the large subunit, the tRNA and the messenger RNA because of its size appeared not to be amenable to any conventional neutron scattering study. In collaboration with K. Nierhaus, MPI Berlin, a programme was defined that is aimed at in situ structure determination of those parts of the ribosome that are of functional interest using nuclear spin contrast variation. The term in situ means that the component of interest is in its native environment. The experiments of polarized neutron scattering were done at the reactor of the GKSS Research Center, Geesthacht.

The preparation of perdeuterated ribosomes is costly, and preparation of the functional complex with its protiated tRNA and well defined stages of translation is tricky. The reader interested in the synchronization of the functional complex for nearly all ribosome molecules of a sample may consult Wadzack et al. [31].

The analysis of the data at that time merged the known structural information from electron microscopy with that from neutron scattering using equ. 24 (Figure 5). Thus the location of the two tRNAs were determined with respect to the ribosome model which had been used. The tRNAs were found to be at the interface between of the two ribosomal subunits. This was not unexpected and it showed that the method of spin contrast variation had been successful. Similarly the line connecting the extremes of the tRNAs with respect to the ribosome could be determined, whereas for the mutual orientation of the planes only a rough estimate could be given [32,33]. The in situ structure and position of a number of ribosomal proteins of the large ribosomal subunit were determined [34].

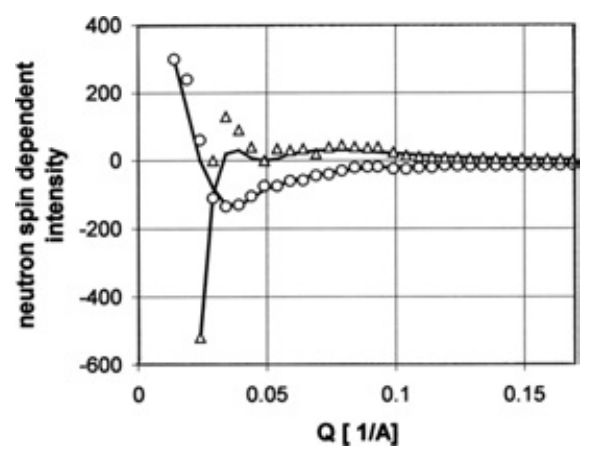

Figure 5. Neutron spin dependent scattering (cross term in equ. 24) of the functional complex of the ribosome from proton polarization $(\mathrm{O})$ and deuteron polarization $(\Delta)$ : The lines are calculated from the model shown in Figure 6. After Nierhaus et al [32]. 


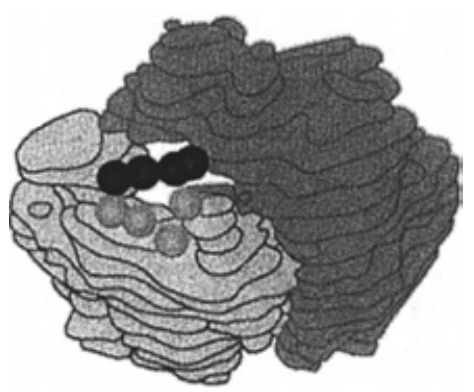

Figure 6. Site and possible orientation of the two tRNAs in the ribosome. The tRNAs are given in a low resolution model of four spheres. The anti codon is close to the neck of the small-subunit (left) whereas the aminoacyl group approaches the central protuberance of the large subunit (right). The diameter of the ribosome is about $270 \AA$ [33]. These results are in good agreement with the model obtained from crystallographic studies using synchrotron radiation [35].

\section{DYNAMIC NUCLEAR SPIN CONTRAST}

By dynamic nuclear contrast we mean a time-dependent nuclear spin contrast, which is observed in timeresolved polarized neutron scattering experiments [12]. As has already been outlined, the evolution of nuclear polarization during DNP occurs at paramagnetic centers. In a second step the more distant nuclei are polarized by spin diffusion. An abrupt, selective change of the polarization will lead to a polarization gradient near paramagnetic centers.

Among the various ways of creating a strong spatial non-equilibrium polarization, the method of AFP seems to be the obvious choice. This method is easily applied for the reversal of the polarization of the bulk protons. It requires a reasonably high initial nuclear polarization that is achieved after prolonged microwave irradiation. The spatial relaxation process after AFP may be much shorter than the time needed for the preparation of the spatial non-equilibrium. In this case only a small fraction of the neutron beam time could be used for the observation of the relaxation. This method was abandoned.

The other way is to address to the protons near the paramagnetic center directly. Again the method of AFP could e considered, but for similar reasons outlined above it has been abandoned as well. The promising alternative is a periodic change in the direction of DNP. This is most easily done by changing the microwave frequency, e.g. from 69.9 to $70.1 \mathrm{GHz}$ (Figure 7).

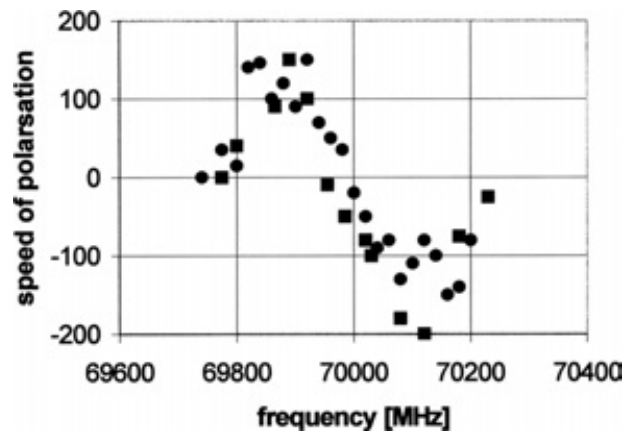

Figure 7. The direction of the speed of dynamic proton polarization (arbitrary units) as a function of the wavelength in the presence of tyrosyl radicals $\left(c=10^{18} \mathrm{~cm}^{-3}\right)$ in a glycerol water mixture. Spheres and squares refer to different scans. $\mathrm{B}=2.5 \mathrm{~T}$. T $=1.2 \mathrm{~K}$. 
The experiments of dynamic nuclear spin contrast variation were done with a DNP facility from PSI. For time-resolved neutron scattering experiments, this set-up was temporarily installed at the instrument D22 of the Institut Laue-Langevin. The neutron experiments on the biradical were done at PSI. Common features of these experiments are

1. The direction of polarization was changed each 5 or $10 \mathrm{~s}$

2. 200 to 400 neutron scattering intensity spectra were collected during one cycle, corresponding to a time resolution of $100 \mathrm{~ms}$ and $50 \mathrm{~ms}$ respectively.

3. The cycle was repeated some hundred to some thousand times

4. The temperature was kept at $1.2 \mathrm{~K}$, the magnetic field was $3.5 \mathrm{~T}$ in most cases and less frequently $2.5 \mathrm{~T}$.

The aim of these experiments is to track down the the propagation of proton polarization during DNP. For this purpose several parameters have been varied:

1. The size of the radical carriers, from the small compact EHBA-Cr(V), over the long rodlike biradical to a relatively large protein, catalase

2. The deuteration of the solvent (EHBA-Cr(V))

3. The concentration (EHBA-Cr(V) and catalase).

\subsection{EHBA-Cr(V)}

Among the radicals supporting DNP, the bis(2-hydroxy-2-ethylbutyrato) oxochromate anion (abbreviated as EHBA-Cr(V)) belongs to the most successful ones. This was one of the reasons why this radical molecule has been chosen for a more detailed study of the mechanism of DNP. Its structure is shown in Figure 8. Another reason is that this molecule can be studied in solvents of different proton concentration. The proton density of the solvent is decreased by addition of heavy water and deuterated glycerol in equal amounts.

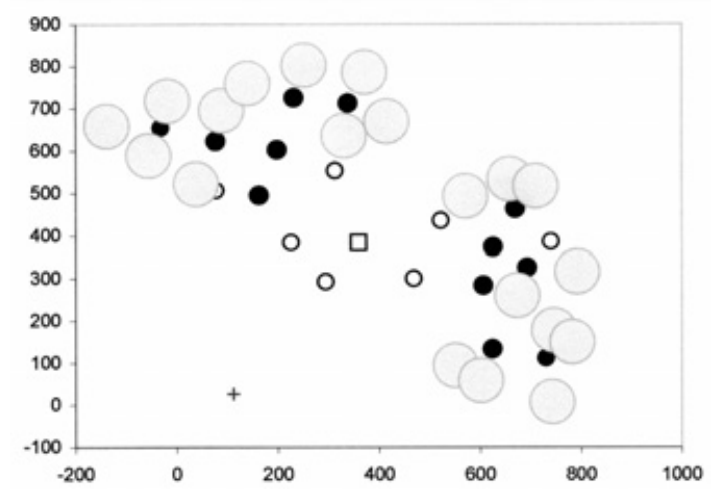

Figure 8. The molecular structure of EHBA-Cr(V) [36]. the hydrogen atoms (big grey spheres) are at distances between 3 and $5 \AA$ from the central chromium atom (square). Oxygen atoms (O), carbon atoms $(\bullet)$.

Time-resolved neutron scattering data have been obtained from EHBA-Cr(V) in solvents with different deuteration. The results are most impressive with EHBA-Cr(V) in a deuterated solvent $(0.98 \mathrm{D})$ (Figure 9). 


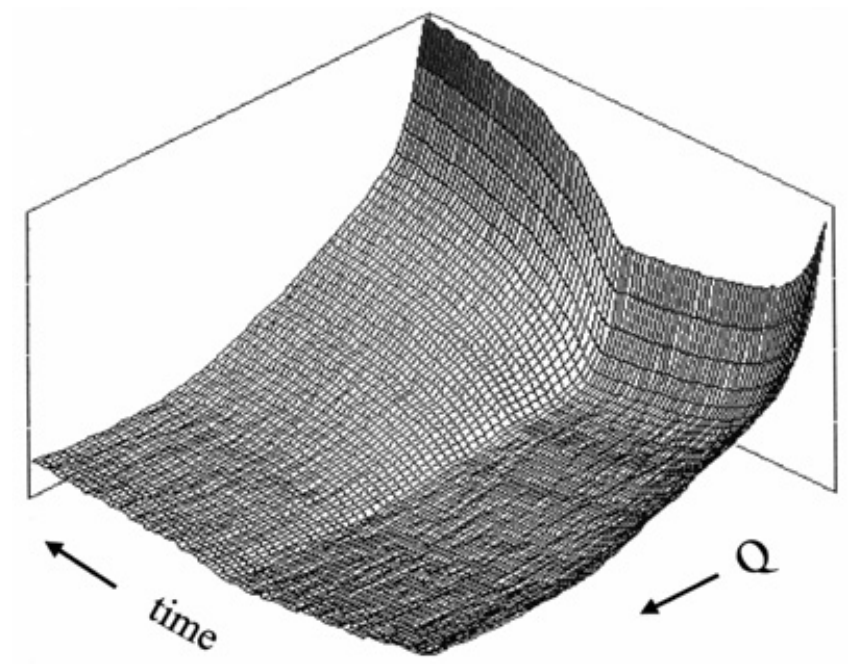

Figure 9. Time-resolved neutron scattering from $\operatorname{EHBA}-\mathrm{Cr}(\mathrm{V})$. The direction of polarization was changed each $10 \mathrm{~s}$, triggering synchronously the aquisition of neutron scattering intensity spectra each $100 \mathrm{~ms}$. During a full cycle of positive and negative DNP 200 spectra were measured. 200 cycles have been averaged. The Q-range extends from 0.03 to $0.7 \AA^{-1}$. The sample contains $5 \times 10^{19}$ paramagnetic centers $/ \mathrm{cm}^{3}$.

The scattering length density of the solvent is very weakly dependent on polarization, so that the change of the coherently scattered neutron intensity with time is almost entirely due to the polarization of the 20 protons of the EHBA-Cr(V) molecules. These can roughly be modelled as a shell of $\mathrm{C}_{2} \mathrm{H}_{5}$ residues surrounding the $\left[\mathrm{CrO}_{7} \mathrm{C}_{4}\right]^{-}$core (Figure 8) [12].The data of the 200 time frames have been fitted with this model assuming the following:

1. A constant structure of the complex.

2. A spatially homogeneous but time-dependent polarization for the protons of the complex

3. A Q-independent but time dependent incoherent scattering.

The first two assumptions mean that the spin diffusion barrier lies outside the EHBA molecule. A crude estimate yields $8 \AA$ for the radius of an isotropic diffusion barrier. This is the distance at which the difference in the z-component of the field created by the paramagnetic center at neighboring nuclei is comparable with the bulk NMR line width. The field gradient is of the order $3 \mu_{B} / r^{4}$, which gives 250 $\mathrm{G} \AA^{-1}$ at $r=5 \AA$, roughly outside the EHBA-Cr(V) molecule [9]. From the variation of contrast with time one can deduce the time dependence of the polarization of the close protons, i.e. the protons of the radical molecule (Figure 10). It can be fitted to the sum of two exponentials. $\tau_{1}=1.1 \mathrm{~s}$, and $\tau_{2}=5.5 \mathrm{~s}$. The bulk polarization measured simultaneously by NMR is also shown in Figure 10.

The difference in the time-evolution of the polarization between close and bulk protons reflects the mechanism of DNP: a strong initial gradient develops due to the fast polarization of the protons close to the paramagnetic center that then spreads out to the bulk with a slower rate [9].

This behavior suggests an interpretation in rate equations describing a flow of polarization between three thermal reservoirs coupled in series, which is meant to explain the time-resolved neutron scattering data from EHBA-Cr(V) in less deuterated solvent. The reservoirs are identified as follows : the electronic spin-spin interaction reservoir, R0, is cooled by the microwaves and acts as a 'source' of polarization. The 20 protons of the EHBA-Cr(V) molecule constitute the reservoir R1,coupled to the source, and the 


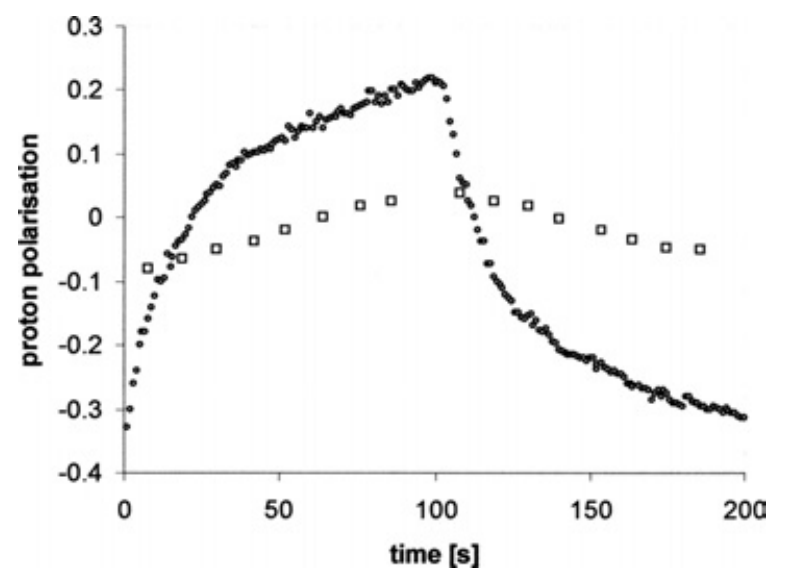

Figure 10. Close proton polarization deduced from the fit of the neutron scattering data (circles) and bulk proton polarization recorded by NMR (squares). The fit obtained by the sum of two exponentials uses the same weights (with opposite sign) and time constants $\left(\tau_{1}=1.1 \mathrm{~s}, \tau_{2}=5.5 \mathrm{~s}\right)$ for the two parts of the evolution of close proton polarization.

bulk protons (those of the solvent) form a reservoir R2, coupled to R1. Two rate equations govern the dynamics.

$$
\begin{gathered}
\frac{d P_{1}}{d t}=\frac{W_{01}}{N_{1}}\left(P_{0}-P_{1}\right)-\frac{W_{12}}{N_{1}}\left(P_{1}-P_{2}\right) \\
\frac{d P_{2}}{d t}=\frac{W_{12}}{N_{2}}\left(P_{1}-P_{2}\right)
\end{gathered}
$$

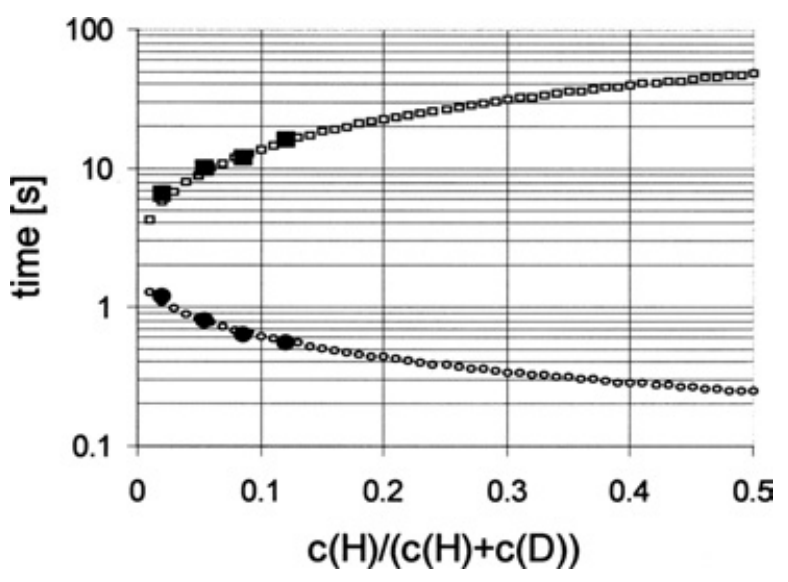

Figure 11. The time constants deduced from the fit to the measured neutron scattering intensities for four solutions of EHBA-Cr(V) in solvents of various degrees of deuteration.

$N_{i}$ and $P_{i}$ denote the number and polarization of protons belonging to the reservoir $\mathrm{Ri}$, and $P_{0}$ denotes the polarization of the source ( +1 during positive DNP and -1 during negative DNP). The rate constants $W_{i j}$ are defined as probabilities of a mutual spin flip per time unit. $W_{01}$ characterizes the flow 
of polarization from $\mathrm{R} 0$ to $\mathrm{R} 1$, and $W_{12}$ characterizes the coupling of the reservoirs $\mathrm{R} 1$ and $\mathrm{R} 2$. For the sample with 0.98 deuteration of the solvent, the unknown values of $W_{01}$ and $W_{12}$ are adapted so that the measured $\tau_{1}$ and $\tau_{2}$ coincide with the time constants of the model solutions. In order to extrapolate these time constants to higher bulk proton concentration, $c_{b u l k}$, we assume that $W_{01}$ to be concentration independent and we make the ansatz $W_{12}=\left(c_{\text {bulk }}\right)^{\beta}$. The best fit to the experimental data is found to be $\beta=0.8$, as shown in Figure 11. The long constant, $\tau_{2}$, essentially describes the built-up of the bulk proton polarization. It is slowed down with increased bulk proton concentration due to the heat capacity of R2 being proportional to $c_{b u l k}$. The dependence of the short time constant, $\tau_{1}$ is largely due to a better coupling between the reservoirs R1 and R2 [37].

Time-resolved neutron scattering data from EHBA-Cr(V) have also been taken with solvents less deuterated than shown in Figure 11. The analysis of these data is more difficult because of the lower contrast of the solute and the increased intensity of incoherent scattering.

The built-up of polarization in samples with lower concentration of EHBA-Cr(V) is considerably slowed down, as the electron spin-spin interaction reservoir becomes less efficient [38].

\subsection{A biradical}

The biradical (Figure 12) has been synthesized for the purposes of EPR spectroscopy [39]. The distance between the radicals on the nitroxide groups is $38 \AA$. The interest in this compound is evident in the light of the assumptions made for analysis of the data from EHBB-Cr(V), notably the assumption of a spatially homogeneous built up of proton polarization during DNP. In view of the large distance of the radicals from hexyl rich core of the biradical, the prediction is permitted that the polarization during DNP might be characterized by an intramolecular gradient. This experiment therefore might add new elements for the understanding of DNP [40] DNP experiments showed that a nuclear polarization of 0.6 could be obtained with a spin lattice relaxation time of $T_{1}=200 \mathrm{~s}$ at $\mathrm{B}=3.5 \mathrm{~T}$ and $\mathrm{T}=1.2 \mathrm{~K}$. The experiments of time-resolved polarized neutron scattering were done on the instrument SANS-1 of SINQ with a static magnetic field of $2.5 \mathrm{~T}$ and at a temperature of $1.2 \mathrm{~K}$. The neutron scattering experiment followed nearly the same protocol which has been used with EHBA-Cr(V), except for the number of spectra which was doubled to 400 per cycle of $20 \mathrm{~s}$ duration. The average from 2000 cycles was analyzed in a slightly more
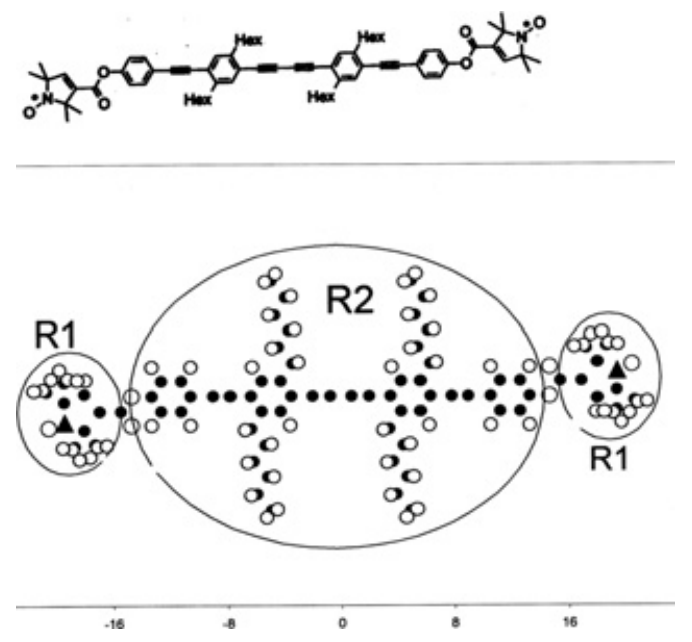

Figure 12. The structure of the biradical. 
generalized mathematical formalism. In order to track down an eventual intramolecular polarization gradient the number of reservoirs was increased. Their rate equations are.

$$
\begin{gathered}
\frac{d P_{1}}{d t}=\frac{W_{01}}{N_{1}}\left(P_{0}-P_{1}\right)-\frac{W_{12}}{N_{1}}\left(P_{1}-P_{2}\right) \\
\frac{d P_{2}}{d t}=\frac{W_{12}}{N_{2}}\left(P_{1}-P_{2}\right)-\frac{W_{23}}{N_{2}}\left(P_{2}-P_{3}\right) \\
\frac{d P_{3}}{d t}=\frac{W_{23}}{N_{3}}\left(P_{2}-P_{3}\right)
\end{gathered}
$$

The meaning of the expressions is analogous to that in (21). The protons of the solute are now in two different reservoirs R1 and R2, and those of the solvent belong to R3. The solutions $P_{1}(t), P_{2}(t)$ and $P_{3}(t)$ of the three differential equations are found by numerical methods.

The protons in $\mathrm{R} 1$ and $\mathrm{R} 2$ of the biradical give rise to the scattering amplitudes $V 1(\mathbf{Q})$ and $V 2(\mathbf{Q})$. The scattering amplitude $U(\mathbf{Q})$ is effective in the absence of nuclear polarization. The total timedependent scattering amplitude is

$$
A(\mathbf{Q})=U(\mathbf{Q})+P_{1}(t) V_{1}(\mathbf{Q})+P_{2}(t) V_{2}(\mathbf{Q})
$$

The expansion of the amplitude as a series of spherical harmonics provides an elegant short-cut to the scattering intensity from randomly oriented biradical molecules. As an example, the coefficient $U_{l, m}$ of $U(\mathbf{Q})$ is

$$
U_{l, m}=\frac{1}{4 \pi} \int U(\mathbf{Q}) Y_{l, m}(\Omega) d \Omega=\sqrt{\frac{2}{\pi}} \sum_{k} b_{k} j_{k}\left(Q r_{k}\right) Y_{l, m}^{*}\left(\omega_{k}\right)
$$

where $Q=|\mathbf{Q}|=(4 \pi / \lambda) \sin \vartheta$, with $\lambda=$ wavelength and $2 \vartheta=$ scattering angle. $\Omega$ and $\omega$ are unit vectors in momentum space and real space, respectively. The k-th atom with the scattering length $b_{k}$ has the polar co-ordinates $\left[r_{k}, \omega_{k}\right]=\left[r_{k}, \Theta_{k}, \phi_{k}\right] ; j_{l}$ are the spherical Bessel functions, and $Y_{l, m}$ are the spherical harmonics. The scattering intensity then is

$$
I(Q)=\left\langle|U(Q)|^{2}\right\rangle=2 \pi^{2} \sum_{l=0}^{l=L} \sum_{m=-l}^{m=l}\left|U_{l, m}(Q)\right|^{2}
$$

The brackets $\langle\ldots\rangle$ denote the average over all orientations of the dissolved particle. The time dependence of coherent polarized neutron scattering is

$$
I(Q, t)=\left\langle|U(\mathbf{Q})|-S(\mathbf{Q})+P 1(t) V 1(\mathbf{Q})+\left.P 2(t) V 2(\mathbf{Q})\right|^{2}\right\rangle
$$

$\mathbf{S}(\mathbf{Q})$ is the scattering amplitude of the shape of the biradical molecule, which takes into account that the biradical is embedded in deuterated polystyrene. The analysis of $I(Q, t)$ from the experiment used its time dependent part only. For this purpose the time-average of $I(Q, t)$ has been subtracted for each Q-interval.

$$
I^{\prime}(Q, t)=I(Q, t)-I_{a v}(Q)
$$

This functions includes the time-dependent parts of coherent scattering, incoherent scattering and background scattering. While the incoherent scattering is easily recognized from its independence from $\mathrm{Q}$, 
this is less so for the separation of coherent scattering from the biradical and the background scattering, as the latter is not directly accessible in an experiment [40].

Both models, as defined in (21)(two-reservoir model,R0 is not counted) and (22)(three-reservoir model) were compared with the experimental data. In a least squares fit the $\chi$-values from the twoand three-reservoir model are 1.8 and 1.6, respectively. A clearer advantage of the three-reservoir model comes from the condition I' $(\mathrm{Q}, \mathrm{t})=0$, which defines a function $\mathrm{Z}(\mathrm{Q})$ (Figure 13).

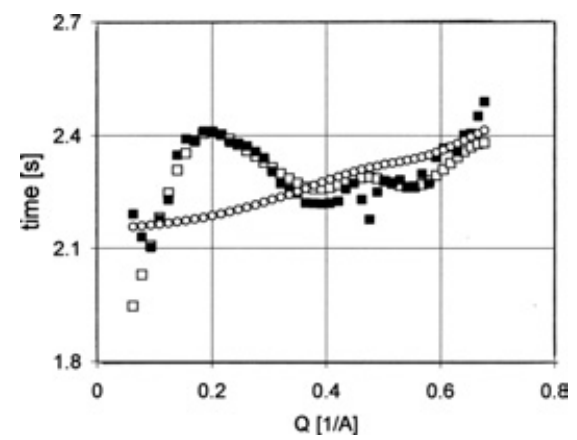

Figure 13. The function $Z(Q)$ from the experimental data (squares) and from the models : Two-reservoirs (circles) and three reservoir (open squares).

At this point,the three-reservoir model with its non-uniform built-up of proton polarization during DNP is to be preferred to a model starting from a spatially homogeneous polarization built-up. Sofar the boundary of R1 of the the three-reservoir model has been sphere with $r=4.2 \AA$. R1 will now be increased at the cost of $\mathrm{R} 2$ in steps of $1.5 \AA$. This changes neither the $\chi$ value of 1.6 nor the good fit of the calculated $\mathrm{Z}(\mathrm{Q})$ with that from the experiment as long as $\mathrm{r}$ is smaller than $12 \AA$. With a further increase of R1 $\chi$ approaches abruptly 1.8, the value from the two-reservoir model and the the $\chi$ value from the fit of $\mathrm{Z}(\mathrm{Q})$ changes from 0.4 to 0.9 . Hence, the three-reservoir model is valid even with an enlarged R1. A magnetic spin diffusion barrier might be extend to the radius of $r=10$ Åapproximately, as predicted [9]. The data analysis also shows that there is hardly any dipolar interaction of the protons of R1 with those in the deuterated polystyrene matrix. The only escape of proton polarization is along the axis of the biradical, which also could reduce the contact between R1 and R2. The evolution of proton polarization in the reservoirs R1, R2 and R3 is shown in Figure 14.

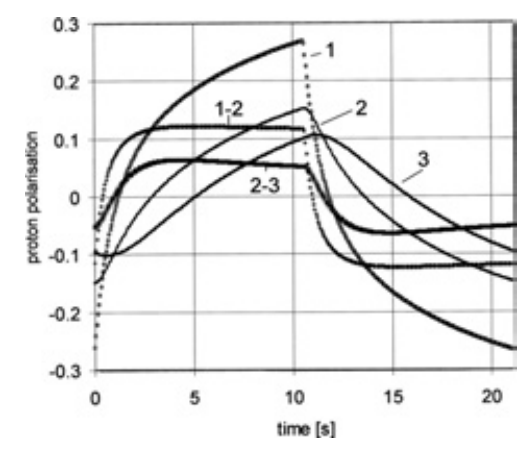

Figure 14. The built-up of proton polarization in the reservoirs R1, R2, and R3 of the three-reservoir model during a cycle of positive and negative polarization. The differences in the proton polarization in the reservoirs R1/R2 (1-2) and R2/R3 (2-3) reach nearly final values within 1 s and 2 s, respectively. 


\subsection{Catalase}

One might believe free radicals are not high in nature's favor as living cells do their best to defend themselves against these. Nevertheless they are important in certain enzymes central to energy transduction in biology. For instance, some steps in photosynthesis involve the formation of radical intermediates. The number of enzymes known to develop a radical state of functional importance at one of its amino acids is rapidly growing due to more sophisticated methods of EPR [41].

Catalases are redox enzymes responsible for the decomposition of hydrogen into water and oxygen. These metalloproteins can be found in aerobic organisms and play a crucial role in cell detoxification. The crystal structures of eight haem catalases have been solved. All of them are homotetramers. Each of the four subunits has an active site near the iron atom of the heme group [42].

The heme active site of the native catalase is in a high spin ferric state $\left(\mathrm{Fe}^{3+}\right)$, i.e. the d electron spins are largely parallel. It can convert to an intermediate state due to two-electron oxidation by hydrogen peroxide. One electron is removed from the iron atom, which thus forms the oxoferryl moiety $\left(\mathrm{Fe}^{4+}=\mathrm{O}\right)$ with the oxygen from the hydrogen peroxide molecule. The second electron is removed from the porphyrin, resulting in $\pi$-cation radical. There is a different response when hydrogen peroxide is replaced by a closely related substance, the peroxyacetic acid. EPR measurements have shown that a tyrosyl radical is formed when catalase from bovine liver is treated with peroxyacetic acid $[43,44]$. Spin quantification yields 0.8 spin per heme. Moreover a detailed analysis of EPR has shown that the tyrosine at position 369 of the amino acid sequence has taken on a radical state [44].

Although no functional role is assigned to tyrossyl-369, the product is an interesting case for a first study of dynamic nuclear contrast variation in a protein. Tyrosine-369 inside the particle of $120 \AA$ diameter finds itself at only $16 \AA$ from the center of the catalase molecule. We recall, that each of the four subunits of catalase can have its tyrosine-369 transformed into radical state. The distance between tyrosine-369 and the heme group is $14 \AA$ (Figure 15).

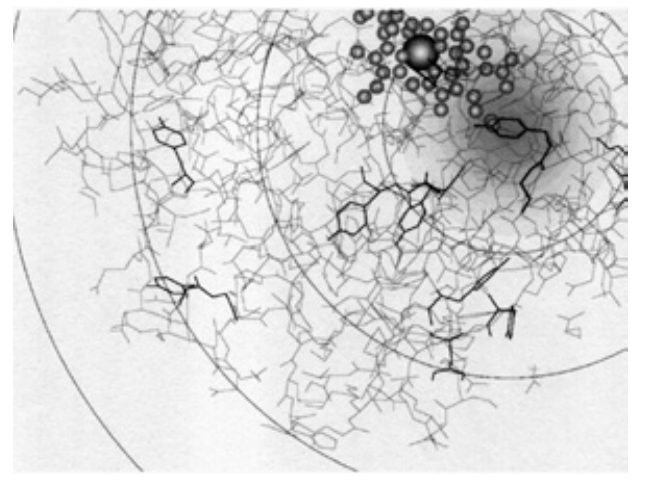

Figure 15. Catalase. One quarter of the structure [42] is shown together with the tyrosine residues (bold lines). The hypothetical size of the proton polarization domain around tyr-369 is marked by a halo. The spheres are atoms of the heme group.

In principle the co-ordinates of the unpaired electron of the radical with respect to the catalase molecule can be determined by magnetic neutron scattering. The expected relative change of the scattering intensity at small angles due to the interaction of the neutron spin with the spin of unpaired electron is $10^{-4}$, probably too small to be detected using magnetic neutron scattering. A promising alternative is to polarize protons close to the unpaired electrons of the multi-radical system that catalase is, as it has been shown for the biradical, which showed an increase of the scattering length in R1 with respect to R2 that was 15 times the magnetic scattering length of an unpaired electron. 
Time-resolved neutron scattering experiments were done in the same way as for EHBA-Cr(V) and the biradical, except for the length of the period, which was halved to $5 \mathrm{~s}$ of DNP : The number of cycles was increased to 2500 . Three samples with 7,15 and $31 \mathrm{~g} / \mathrm{l}$ catalase were measured. The radical concentrations were 65,200 and $300 \mu \mathrm{Mdm}^{-3}$ corresponding to $0.33 \times 10^{17}, 1.2 \times 10^{17}, 1.8 \times 10^{17}$ unpaired electrons $/ \mathrm{cm}^{3}$. This is two orders of magnitude less than with the solutions of EHBA-Cr(V) and the biradical. The relative change of the intensity was $1.8 \times 10^{-3}$ at $Q=0.026 \AA^{-1}$. Figure 16 shows the time dependence of the neutron scattering intensity during one cycle at $Q=0.26$ and 0.52 $\AA^{-1}[24]$.
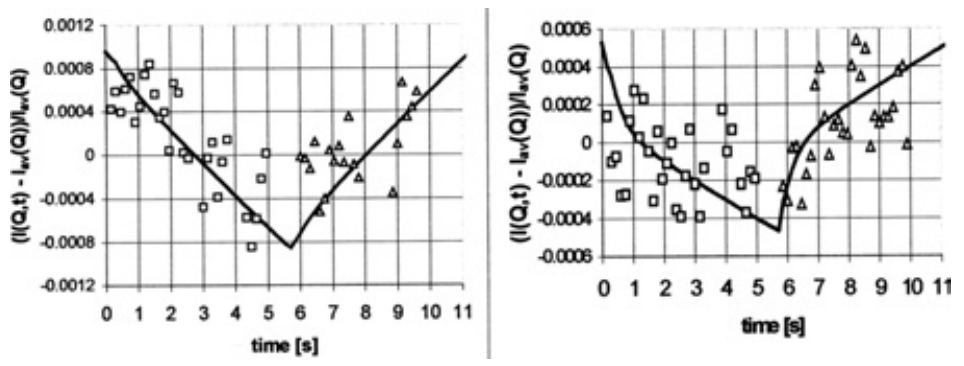

Figure 16. The relative change in the intensity of neutron scattering during DNP at $Q=0.26$ and $0.52 \AA^{-1}$.

The three-reservoir model which has been successful with the biradical is used for the analysis of the time-resolved neutron scattering data of catalase. R1 is defined by the protons within a sphere of 20 A diameter centered at the tyrosine-369. All the other protons of catalase belong to R2. The protons of the deuterated solvent constitute R3. The built up of proton polarization in R1 is fast, as it has been observed with the biradal. During a half-cycle of $5 \mathrm{~s}$ about 1.5 protons per tyrosyl radical are polarized, quite similar to the biradical. But there are also important differences with respect to the biradial. The proton polarization of catalase remains always positive, somewhat below the value of proton polarization reached at thermal equilibrium, i.e. 0.0035 for $\mathrm{B}=3.5 \mathrm{~T}$. Prolonged negative DNP lowers the proton polarization to about half this value. The number of polarized protons in R2 (Figure 17) corresponds to about $2 / 3$ of the proton polarization at thermal equilibrium.

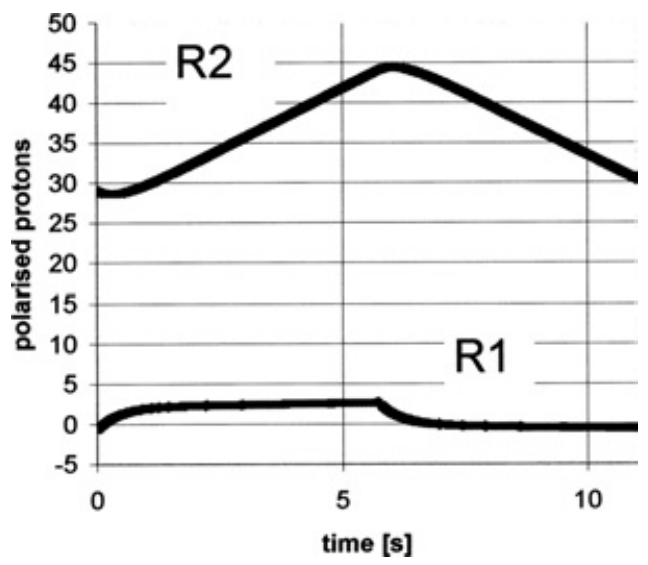

Figure 17. The built-up of proton polarization in the reservoirs R1 (protons close to Tyr-369) and R2(other protons of catalase) of the three-reservoir model during a cycle of positive and negative polarization [24]. 
There are 20 tyrosines per subunit of the catalase molecule. Assuming that each of these could have been transformed into the radical state, the fit of the scattering intensity calculated from the model with the experimental data has been done for all possible tyrosyl radical sites separately. There is fairly good agreement for those tyrosins which are relatively close to the center of the catalase molecule. Tyrosine369 belongs to these but all those within a distance of $40 \AA$ from the center meet this condition as well. The accuracy of the present data (Figure 16) does not allow a more precise localization of the radical site [45].

\section{CONCLUSION}

Nuclear spin contrast variation is very much driven by applications in macromolecular structure research. This is evident from the results obtained from polymers and biological molecules. A closer inspection of the first experimental results has lead to new questions concerning the mechanism of the proton polarization built-up. The experimental evidence of the proton spin diffusion barrier in a protiated system from polarized neutron scattering remained inaccessible until recently. Both the creation of intramolecular proton polarization gradients by the methods of dynamic spin contrast and the high proton polarizations needed for static spin contrast, open new ways in the use of neutrons for science.

\section{References}

[1] Hayter, J.B., Jenkin, G.T., White, J.W., Phys. Rev. Lett. 33, 696 (1974).

[2] des Cloizeaux, J., Jannink, G., Les Polymères en Solution. leur Modélisation et leur Structure, les éditions de physique, France. Les Ulis (1987).

[3] Leslie, M., Jenkin, G.T., Hayter, J.B., White, J.W., Cox, S., Warner G., Phil. Trans. R. Soc. B 290, 497 (1980).

[4] Motoya, K., Nishi, M., Itoh, Y., Solid State Commun. 33, 143 (1980).

[5] Knop et al, W., Helvetica Physica Acta, 50, 741 (1986).

[6] Knop et al, W., Appl.Cryst. J., 24,493 (1991).

[7] Koghi, M., Ishida, M., Ishikawa, Y., Ishimoto, S., Kanno,Y., Masaike, A., Masuda, Y., Moromoto, K., J. Phys. Soc. Japan 56,2681 (1987).

[8] Glättli, H., Fermon, C., Eisenkremer, M., Pinot, M., Physique, J., 50, 2375 (1989).

[9] van den Brandt, B., Europhys. Lett. 59, 62 (2002).

[10] Abragam. A.,and Goldman, M., Nuclear Magnetism : Order and Disorder, Oxford : Clarendon (1982).

[11] Glättli, H., Goldman, M., Methods Exp. Phys. C 23, 241 (1987).

[12] Leymarie, E., Thesis, Université Paris XI Orsay, No7052 (2002).

[13] Ohlsen, G.G., Rep. Prog. Phys. 35,717 (1972).

[14] Overhauser, A.W., Phys. Rev. 92, 411 (1953).

[15] Abragam, A., Phys. Rev. Rev. 98, 1729 (1955).

[16] Abragam, A., Goldman, M., Rep. Prog. Phys. 41, 395 (1978).

[17] Goertz, St., Meyer, W., Reicherz, G., Prog. Part. Nucl. Phys. 49,403 (2002).

[18] Borghini, M., Phys. Rev. Lett. 20, 419 (1968).

[19] Goldman, M., Fox, S.F.J., Bouffard, V., J.Phys. C: Solid State Phys. 7, 2940 (1974).

[20] Cox, S.F.J., Read, S.F.J., Wenckebach, Th., J. Phys. C : Solid State Phys. 10, 2917 (1977).

[21] Wenckebach, Th., Proc. 2nd Workshop on Polarised target Materials, Rutherford and Appleton Labs. RL-80-080

[22] Niinikoski T.O., Proc. 2nd Workshop on Polarised target Materials, Rutherford and Appleton Labs. RL-80-080 
[23] Abragam, A., The Principles of Nuclear Magnetism, Oxford : Oxford University Press.

[24] Stuhrmann, H.B., Rep. Prog. Phys. 1073-1115 (2004).

[25] van den Brandt et al. B., AIP Proc. 187, 1251 (1989).

[26] van den Brandt, B., Hautle, P., Konter, J.A., S. Mango PSI Sci.Rep. 2000 III, 78 (2001).

[27] Niinikoski, T.O., Nucl. Instrum. Methods Phys. Res. A 356, 62 (1995).

[28] Hautle, P., Grübler, W., van den Brandt, B., Konter, J.A., Mango, S., M. Wessler, Phys. Rev. B 46 6596 (1992).

[29] Krumpolc, M., Rocek, J., J. Am. Chem. Soc. 1013206 (1979).

[30] M.S. Capel et al. Science 238, 1403.

[31] J. Wadzack et al. J. Mol. Biol. 266, 343 (1997).

[32] Nierhaus, K.H., Wadzack, J., Burkhardt, N., Jünemann, R., Meerwinck, W., Willumeit, R., Stuhrmann, H.B., Proc. Nat.Acad. Sci. USA 95, 945-950 (Biochemistry) (1998).

[33] Stuhrmann, H.B., Nierhaus, K.H., Neutrons in Biology Vol 397, ed. Schoenborn and Knott, New York : Plenum (1996).

[34] Willumeit, R., Diedrich, G., Forthmann, S., Beckmann, J., May, R., Stuhrmann, H.B., Nierhaus, K.H., Biochim. Biophys. Acta 15207 (2001).

[35] F. Schlünzen et al. Cell 102,615 (2000).

[36] Krumpolc, M., DeBoer, B.G., J Rocek, J. Am. Chem. Soc. 100, 145.

[37] B. van den Brandt et al. Physica B 335, 193.

[38] van den Brandt, B., Glättli, H., Grillo, I., Hautle, P., Jouve, H., Kohlbrecher, J., Konter, J.A., Leymarie, E., Mango, S., May, R.P., Michels, A., Stuhrmann, H.B., O. Zimmer, Eur. Phys. J. B49, 157-165 (2006).

[39] Godt, A., Franzen, C., Veit, S., Enkelmann, V., Jeschke, G., J. Org. Chem. 65, 7575-7582 (2000).

[40] van den Brandt, B., Glättli, H., Godt, A., Hautle, P., Kohlbrecher, J., Konter, J.A., Mango, S., Michels, A., Stuhrmann, H.B., O. Zimmer (manuscript in preparation).

[41] Faller, P., Goussias, C., Rutherford, A.W., S. Un, Proc. Nat. Acad. Sci. USA, 100, 8732.

[42] Bravo, J., Fita, I., Gouet, P., Jouve, H.M., Meli-Adamyan, W., Murshukov, G.N., Structure of Catalase in Oxidative Stress and the Molecular Biology of Antioxidants Defences, ed. J.G. Scandalios, New York : Cold Spring Harbor Press Laboratory Press, pp 407-445 (1997).

[43] Ivancich, A., Jouve, H.M., J. Gaillard, Biochemistry 36, 9356.

[44] Andreoletti, P., Gambarelli, S., Sainz, G., Stojanoff, V., White, C., Desfonds, G., Gagnon, G., Gaillard, J., Jouve, H.M., Biochemistry 40, 13734 (2002).

[45] van den Brandt, B., Gaillard, J., Glättli, H., Grillo, I., Hautle, P., Jouve, H., Kahn, R., Kohlbrecher, J., Konter, J.A., Leymarie, E., Mango, S., May, R.P., Stuhrmann H.B., and O. Zimmer (manuscript in preparation). 